\title{
Wavelet Analysis of Vibration Signals Part 2: Wavelet Applications
}

\author{
David E. Newland \\ Department of Engineering, University of Cambridge, Trumpington Street, Cambridge CB2 1PZ, England \\ e-mail:den@eng.cam.ac.uk
}

\begin{abstract}
This is the second of a pair of papers on the application of wavelet theory to vibration analysis. For transient data analysis, the wavelet transform and the short-time Fourier transform (STFT) produce similar results, but wavelet transforms have computational and other advantages which may be important in particular applications. This part of the paper discusses the construction of time-frequency maps. It is shown how the harmonic wavelet transform, HWT, may be modified to improve the clarity of a time-frequency map and an example is given in which a map computed by the lapped HWT is compared with a corresponding map computed by the STFT.

It is found that the windowed HWT is good for time-frequency mapping because of its flexibility and ease of use and the computational efficiency of its algorithm.
\end{abstract}

\section{MEAN-SQUARE WAVELET MAPS}

For harmonic wavelets, wavelet level defines a finite frequency band; for dilation wavelets, wavelet level is a measure of the center frequency of a localized but infinite band of frequencies. Therefore wavelet level is interchangeable with frequency for harmonic wavelets and a measure of predominant frequency for dilation wavelets.

The mean-square map depends on the result that the mean-square value can be represented as a summation of the magnitudes of the wavelet coefficients squared. For discrete dilation wavelets, the summation is

$$
\frac{1}{2^{n}} \sum_{r=0}^{2^{n}-1} x_{r}^{2}=a_{0}^{2}+\sum_{j=0}^{n-1} 1 / 2^{j} \sum_{k=0}^{2^{j}-1} a_{2^{j}+k}^{2}
$$

while, for harmonic wavelets, it is

$$
\begin{gathered}
\frac{1}{2^{n}} \sum_{r=0}^{2^{n}-1} x_{r}^{2}= \\
a_{0}^{2}+\sum_{j=0}^{n-2} 1 / 2^{j} \sum_{k=0}^{2^{j}-1}\left\{\left|a_{2^{j}+k}\right|^{2}+\left|a_{2^{n}-2^{j}-k}\right|^{2}\right\}+a_{N / 2}^{2}
\end{gathered}
$$

for the corresponding case when the frequency blocks cover octave bands.

If a three-dimensional graph is constructed with the moduli of wavelet amplitudes squared plotted on a base plane whose axes are wavelet level (represented by integer $j$ ) in one direction and position (represented by integer $k$ ) in the other (orthogonal) direction, the volume under the resulting surface can be normalised to be a measure of mean-square. Where there are significant contributions to a signal's energy at particular frequencies and times, the mean-square surface will have localized peaks. Therefore a contour plot of the mean-square surface will generate a two-dimensional map which illustrates the distribution of energy (i.e. mean-square value) over frequency and position.

Two examples are shown in Fig. 10. Wavelet maps of the same length of band-limited signal (shown) are drawn (a) for harmonic wavelets and (b) for dilation wavelets with $N=20$ wavelet coefficients. The signal is represented by the discrete function $x_{r}, r=0$ to 511 , and the corresponding wavelet maps are shown immediately below this signal. Also, for clarification, mesh diagrams of the underlying shapes are drawn below each map. In the case of the harmonic wavelet map, it can be seen that the energy of the signal is confined to level 5 of the transform only. This is because $x_{r}$ has been chosen to have a band-limited spectrum which falls within the frequency band of level 5 of the harmonic wavelet transform. For dilation wavelets, the frequency spread is wider and is not band-limited. Therefore energy spreads over several levels of the dilation wavelet map as shown in Fig. 10(b). The spacing of the contours and the vertical scale of the mesh diagrams in Fig. 10 are logarithmic, so the high peaks are relatively diminished in magnitude, but there is general correspondence between the location in time of the high peaks in $x_{r}$ and the high peaks on the wavelet maps.

The use of mean square wavelet maps to identify irregularities in otherwise smooth signals is shown in Figs. 11 and 12. The first shows a signal with a periodic local perturbation - almost invisible to the eye - and the corresponding harmonic wavelet map and mesh diagram with logarithmic scales. The identity and location of the peaks are now visible clearly. In Fig. 12, a harmonic signal has a single local higher-frequency perturbation. The wavelet map and mesh diagram, again with logarithmic scales, allow this to be identified immediately. The identification of small, local perturbations on otherwise smoothly-varying signals is one of the principal applications of wavelet analysis and appears to offer considerable benefits in the field of vibration monitoring.

\section{COORDINATES OF HWT TIME-FREQUENCY MAPS}

